\title{
Concept Analysis of Health Literacy for Patients with Cardiovascular Disease using Hybrid Model
}

\author{
Sim, Jeong Eun ${ }^{1 \oplus} \cdot$ Hwang, Seon Young ${ }^{2}$ \\ ${ }^{1}$ Doctoral Student, Graduate School of Nursing, Hanyang University, Seoul \\ ${ }^{2}$ Professor, School of Nursing, Hanyang University, Seoul, Korea
}

Purpose: The purpose of this study is to provide a clear definition of the health literacy for patients with cardiovascular disease by analyzing the dimensions and properties using Hybrid concept analysis. Methods: The concept of health literacy of patients with cardiovascular disease was analyzed according to the cyclic process of theoretical phase-field work phase-final analysis phase presented in the Hybrid model. We reviewed 26 literatures and conducted in-depth interviews with 13 patients with cardiovascular disease. Results: The concept of health literacy in cardiovascular patients is derived from two dimensions and five attributes. Literacy skills, health information search ability and health information utilization skills were derived as attributes in the individual functional dimension, while active communication with the medical team and utilization of health information support resources were derived at the interrelational dimension. It is defined as the individualized and integrated ability of an individual to explore and utilize the various health information needed to make appropriate health decisions during the chronic course after diagnosis of cardiovascular disease, to communicate proactively with medical staffs and to utilize support resources. Conclusion: This study will contribute to the development and related research of health literacy measurement tools that can be used in cardiovascular nursing practice based on the attributes and indicators of health literacy for patients with cardiovascular disease.

Key Words: Cardiovascular diseases; Chronic disease; Health literacy; Information literacy

\section{INTRODUCTION}

\section{Background}

Cardiovascular disease is the second leading cause of mortality in Korea. The mortality rate from cardiovascular disease rose from $44.0 \%$ in 2007 to $60.2 \%$ in 2017 , increasing by $16.2 \%$ over 10 years. Cardiovascular disease is reported to rank first for healthcare costs among non-infectious diseases, accounting for $16 \%$ of the total healthcare expenditures of non-infectious diseases [1]. In general, cardiovascular disease refers to ischemic heart disease, valvular heart disease, arrhythmia and heart failure. In a broader sense, it also includes underlying chronic diseases, such as hypertension, diabetes mellitus and dyslipidemia [2]. In Korea, the economic burden of cardiovascular dis- ease is expected to continue to increase in the future due to the growth of the older population and a rapid increase in the prevalence of underlying diseases resulting from unhealthy lifestyles [1]. According to the 2014 Korea National Health and Nutrition Examination Survey (KNHANES), the prevalence of cardiovascular disease in Koreans was $31.2 \%$ in 2014 , an increase of $12.2 \%$ compared to 2007 , and the prevalence rate increased with age among Koreans, with $6.1 \%$ in the 30 39 age group, $18.2 \%$ in the $40 \sim 49$ age group, $42.0 \%$ in the 50 59 age group, and $64.7 \%$ in the 60 69 age group [3]. Given the rapid rate of aging in Korea, the prevalence and mortality rate of cardiovascular disease are expected to increase further among community-dwelling patients. Thus, there is a need to develop intervention strategies to actively manage them in terms of primary and secondary prevention.

\footnotetext{
Corresponding author: Hwang, Seon Young

School of Nursing, Hanyang University, 222 Wangsimni-ro, Sungdong-gu, Seoul 04763, Korea.

Tel: +82-2-2220-0702, Fax: +82-2-2220-1163, E-mail: seon9772@hanyang.ac.kr

Received: Aug 1, 2019 / Revised: Oct 30, 2019 / Accepted: Nov 7, 2019

This is an open access article distributed under the terms of the Creative Commons Attribution Non-Commercial License (http://creativecommons.org/licenses/ by-nc/3.0), which permits unrestricted non-commercial use, distribution, and reproduction in any medium, provided the original work is properly cited.
} 
Since the majority of patients with cardiovascular disease depend on regular visits to medical institutions and drug therapy in their lifetime, medication adherence is of the greatest importance for them. The changes and management of risk factors, diet, and lifestyles are essential for prevention of the recurrence or exacerbation of diseases [4]. In particular, as the survival rate increases due to the development of treatment technology, patients with cardiovascular disease are exposed to a large amount of information on complicated and diverse therapeutic methods and health from the medical team. Based on the information, patients with cardiovascular disease acquire health information and learn to take care of their own health on their own over a long period of time, implementing selfcare [5]. Therefore, in nursing practice, it is necessary to accurately assess the health literacy of these patients.

Health literacy is a concept that has recently attracted attention as a determinant of health behavior compliance in patients with cardiovascular disease [6]. It is the ability to understand and utilize health information, and the patient's level of health literacy has been reported to have a significant effect on the patients' prognosis as well as their current health status, including self-care [7]. In Korea, interest in health literacy has been increasing since the preliminary research for the development of Korean health literacy assessment tools was conducted in 2005 [8]. 'Health literacy' has been translated in several different ways in Korea, and Korean translations have literal meanings such as 'medical information comprehension ability', 'health literacy', and 'medical information literacy.' Recently, diverse Korean translations, such as 'health information comprehension ability' or 'health information utilization ability', have been used depending on the nature of research. Some researchers suggest that although literacy and health literacy tend to be used interchangeably in many studies dealing with the association between literacy and health, it is desirable to distinguish two terms from each other. Literacy is a concept related to particular contents and environments. Even though a person has literacy skills that are essential to obtaining, understanding, and utilizing information related to a specific subject, he or she may lack health literacy needed to obtain, understand, and use health information [9]. Therefore, it has been suggested that the two terms should be defined clearly so that people can distinguish them easily and that it is more preferable to use the term 'health literacy' in areas where people can encounter health-related information and messages to prevent confusion about the concepts [10]. In the field of nursing science, research has been conducted to measure the levels of health literacy and identify its influencing factors in patients with coronary artery disease [4], communitydwelling older people [7], hypertensive patients [11], married immigrant women [12], and heart failure patients [13]. In older people, as the level of health literacy became higher, the level of health behavior was increased [7]. In patients with coronary artery disease, health literacy was found to be a predictor of health behavior compliance [4]. It was also shown to be a strong predictor of health behavior compliance in elderly patients with hypertension [11].

Currently, the most widely used health literacy assessment tools in the research of cardiovascular diseases are the Rapid Estimate of Adult Literacy in Medicine (REALM) developed by Davis et al. [14] in 1991 and the Test of Functional Health Literacy in Adult (TOFHLA) developed by Parker et al. [15] in 1995. In Korea, the Korean Health Literacy Assessment Tool (KHLAT) developed by Kim et al. [8], which is a translated and modified version of the REALM, and the 'Korean Health Literacy Measurement Tool' developed by Lee [16], and the Functional Health Literacy Test (KFHLT) developed Kim \& Lee [9] are the most frequently used tools. The REALM and KFHLT are currently health literacy assessment tools close to the gold standard for health literacy measurement. However, they are assessment tools for functional health literacy and their measurement areas are limited to word recognition, reading comprehension, and numeracy of the general population, so the instruments have limitations on the application and interpretations for patients with chronic diseases [10]. Especially, if these tools are applied to patients with cardiovascular disease for whom secondary prevention is essential due to the chronic course of the disease, it is not possible to assess health literacy considering the unique characteristics of cardiovascular diseases. Therefore, it is urgently required to develop a health literacy assessment tool for patients with cardiovascular disease which is suitable for Korean situations and convenient to use for the development of research and nursing practice for patients with cardiovascular disease rapidly increasing in Korea. In particular, identification of the characteristics of health literacy for patients with cardiovascular disease and clarification of the concept needs to be carried out prior to the development of an assessment tool to accurately assess health literacy in patients with cardiovascular disease.

Health literacy can be interpreted as various consequences according to the subjects' culture, lifestyles, and characteristics of individuals' diseases [9]. So far, no generally accepted definition of health literacy taking into account the disease characteristics and cultural characteristics of Korean patients with cardiovascular disease has 
been derived yet, and no clear definition of the concept has been presented. The concept encompasses various meanings, and if researchers do not recognize them correctly, it may give rise to problems with the validity and reliability of research, and these consequences may lead to problems in communication among the researchers [17]. Although the general concept of health literacy has been defined based on a systematic review of the literature in the field of nursing [18], this definition was not derived through prior exploration and accurate understanding of Korean patients with chronic cardiovascular disease, so it is difficult to apply the concept to these patients. Among the methods for concept analysis, the hybrid model is a useful approach when developing a concept which can be applied to the clinical field from the point of view of nursing, because the method allows researchers to analyze a concept which is difficult to apply in the clinical field because of the highly abstract and ambiguous nature of the concept by performing analysis in the fieldwork stage in combination with analysis in the theoretical stage [19]. Therefore, in this study, we intend to conduct a conceptual analysis of health literacy for patients with cardiovascular disease, using Schwartz-Barcott and Kim's hybrid model [19] because it is considered to be an appropriate approach in consideration of the unique propensities and disease characteristics of patients with cardiovascular disease. If the concept of health literacy is clearly defined taking into account the disease characteristics of patients with cardiovascular disease and individuals' illness perception, it is expected to promote related research, including the development of objective measurement tools that can be used in the future nursing practice and research for patients with cardiovascular disease.

\section{Purpose}

The purpose of this study is to present a clear definition of health literacy for patients with cardiovascular disease by analyzing the dimensions and attributes of health literacy for patients with cardiovascular disease through a conceptual analysis using the hybrid model. The specific objectives of this study are as follows.

- To derive the provisional definition of health literacy for patients with cardiovascular disease by identifying the antecedents, consequences, dimensions, and attributes of health literacy for patients with cardiovascular disease through a systematic review of the literature;

- To identify the dimensions, attributes, and indicators of health literacy for patients with cardiovascular dis- ease through field research; and

- To present the dimensions, attributes and indicators of health literacy for patients with cardiovascular disease that can be used in nursing practice and research, based on the attributes of health literacy for patients with cardiovascular disease derived from the theoretical analysis and field research.

\section{METHODS}

\section{Study Design}

This study is a research using conceptual analysis to derive the dimensions, attributes and indicators of health literacy for patients with cardiovascular disease through the three stages of theoretical research, fieldwork, and final analysis.

\section{Procedures and Analysis Methods}

The concept of health literacy for patients with cardiovascular disease was analyzed according to the three-step cyclic process presented in the hybrid model [19].

\section{1) Theoretical stage}

In the theoretical stage of the hybrid model, the dictionary definitions and related literature are examined to investigate the definitions, attributes and essential meanings of a concept. Based on the results, the operational definition of the concept is derived by comparing and analyzing the identified attributes and definitions of the concept [19]. In this study, we examined the dictionary definitions and domestic and foreign academical papers to investigate the essential meanings of health literacy with the aim of identifying the conceptual definitions and attributes of health literacy for patients with cardiovascular disease.

In this study, we conducted literature search of foreign papers published between 2008 and 2018 through the CINAHL (Cumulative Index to Nursing \& Allied Health Literature), PubMed, and EMBASE databases. We used search queries such as 'health literacy or/and cardiovascular disease' and 'health literacy or/and coronary artery disease' as the major search terms and additionally searched papers containing terms such as myocardial infarction, angina pectoris, and heart failure among. As for Korean papers, we searched research papers whose title, keywords, or body contained terms such as cardiovascular disease, myocardial infarction, angina pectoris, coronary artery disease, heart failure, and health literacy or one of the Korean translations for health literacy among the papers 
published during the decade from 2008 to 2018 through the database of the Korea Education \& Research Information Service (www.riss4u.net). Of the 476 retrieved domestic and foreign papers, 69 papers, including duplicates, were initially extracted through the first data extraction process. Then, papers that contained the concept of health literacy were selected by examining the abstracts of the initially extracted studies. Afterwards, studies without the definition of health literacy or those which performed only the assessment of health literacy were excluded. As a result, 26 studies ( 6 domestic and 20 foreign studies) were finally selected, excluding 43 papers that did not deal with both cardiovascular disease and health literacy. Through the analysis of the finally selected studies, the definitions and attributes of the concept of health literacy for patients with cardiovascular disease were extracted and provisional definitions were constructed (Table 1).

\section{2) Fieldwork stage}

In the field work stage of the hybrid model, by analyzing the data collected in the field research, the attributes of the concept identified through the analysis results of the theoretical stage are verified and the newly discovered attributes are identified [19]. In this study, a total of 13 par- ticipants were selected by convenience sampling among patients aged 30 or older who were diagnosed with myocardial infarction, angina pectoris, heart failure, or arrhythmia and were admitted to hospital or were receiving outpatient treatment in the outpatient care unit of the Division of Cardiology, Department of Internal Medicine at $\mathrm{K}$ University Hospital in $\mathrm{S}$ city. The attributes and concepts of health literacy for patients with cardiovascular disease were derived, and they were verified through interviews with the participants. The specific inclusion criteria for the participants were as follows: Adults aged 30 years or older; diagnosed with coronary artery disease, heart failure, arrhythmia, or valvular heart disease; able to read and understand the contents of the questionnaire written in Korean; have no difficulty in communicating with others; understand the study purpose, agree to participate in the study, and sign the informed consent form. The exclusion criteria were as follows: Individuals diagnosed with organic brain disease or mental illness or individuals who do not agree to participate in the study.

This study was conducted after receiving approval from the IRB of the university to which the researcher belonged (IRB No.: HYU-2019-02-013). The interviews with participants were conducted from January to April 2019, and

Table 1. Components of Health Literacy of Patients with Cardiovascular Disease in Theoretical Stage

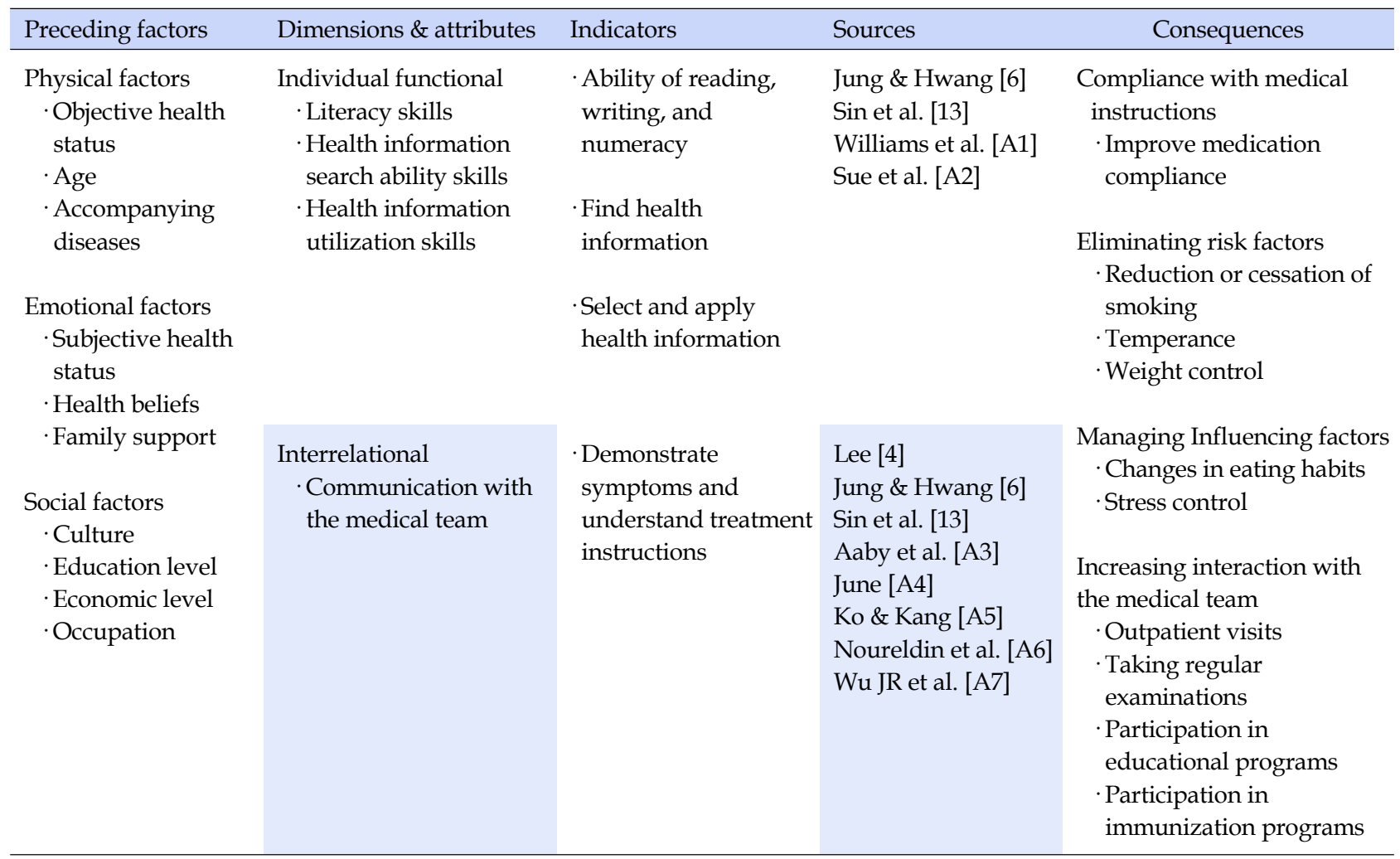

$\mathrm{A}=$ appendix reference. 
they were performed 1 and 2 times per participant, $40 \mathrm{mi}-$ nutes to 1 hour per session. The investigator personally performed in-depth interviews, and they were conducted after obtaining informed consent from participants and explaining to them that they could withdraw from the research at any time if they wanted to. The interviews were carried out using the open-ended questions created after deriving a tentative definition of the concept of health literacy through the theoretical stage. All the contents of the interviews were recorded with the consent of the participants, and their non-verbal expressions were also recorded using field notes.

The major open-ended questions used were as follows: 'How did you normally manage your health?'. 'How much and what do you know about your cardiovascular disease?', 'Where do you usually get information about cardiovascular disease?', 'What kind of difficulty did you have using information about cardiovascular disease?', 'How did you use this information to actually manage your health?', 'What kind of difficulty did you have using medical institutions?', 'What kind of difficulty did you have communicating with your doctor or nurse?', and 'What kind of effort are you currently making to maintain your heart health?' The interviews were carried out in quiet and comfortable places where interviews would not be interrupted, such as an area in the hospital or the participant's residence, and the in-depth interview data derived from the field work stage were analyzed using Strauss and Corbin's grounded theory [20]. According to the proce- dures of grounded theory, we analyzed the interview data through the three coding stages called open coding, axial coding and selective coding. Open coding is the first coding phase for identifying the meaning of each line of the interview contents and labeling it, axial coding is the process of identifying the relationships between the labeled categories by constantly comparing them, and selective coding is the final coding stage to identify the core category among the data categories identified through previous coding phases and link and integrate the major categories around the core category [20]. During the analysis, the investigator used the constant comparison method [20], which is the method of comparing the data with each other and closely examining the similarities and differences, identified the categories generated during the interviews, and continuously conducted interviews until new categories did not emerge any more. All the contents of interviews were transcribed by the investigators. Two investigators derived key concepts and divided the domains based on independently transcribed contents, and they consulted two qualitative research experts during the analysis. The general characteristics of the participants are shown in Table 2.

\section{3) Final analysis stage}

In the final analysis stage, the results of the theoretical and fieldwork stages were compared and analyzed to define the integrated concept of health literacy for patients with cardiovascular disease [19]. By synthesizing the anal-

Table 2. Characteristics of Interviewed Participants

\begin{tabular}{lclllc}
\hline Participants & Age (year) & Gender & Living with & Medical diagnosis & Duration of illness (year) \\
\hline Participant 1 & 75 & Men & Spouse & Myocardial infarction & 2 \\
Participant 2 & 76 & Men & Spouse & Angina pectoris & 4 \\
Participant 3 & 56 & Men & Family & Myocardial infarction & 3 \\
\hline Participant 4 & 48 & Men & Family & Arrhythmia & 1 \\
\hline Participant 5 & 62 & Men & Alone & Heart failure & 1 \\
\hline Participant 6 & 58 & Men & Family & Angina pectoris & 12 \\
\hline Participant 7 & 66 & Men & Spouse & Angina pectoris & 4 \\
\hline Participant 8 & 85 & Women & Family & Heart failure & 2 \\
\hline Participant 9 & 55 & Women & Spouse & Heart failure & 10 \\
\hline Participant 10 & 63 & Men & Alone & Atrial fibrillation & 7 \\
\hline Participant 11 & 78 & Women & Family & Angina pectoris & 8 \\
\hline Participant 12 & 69 & Men & Family & Heart failure & 20 \\
\hline Participant 13 & 83 & Men & Spouse & Myocardial infarction & \\
\hline
\end{tabular}


ysis data according to the contents of each of the major areas identified in the theoretical and fieldwork stages, the attributes and indicators of the concept of 'health literacy of patients with cardiovascular disease' were derived.

\section{RESULTS}

\section{Theoretical Stage}

\section{1) Dictionary definition of health literacy}

The most commonly used Korean translation for health literacy literally means 'health information comprehension ability.' In Korean dictionaries, the term 'health' is generally defined as 'soundness or robustness of the mind and body or the condition of being mentally and physically sound and vigorous', and 'information' is defined as knowledge acquired by organizing facts or data gathered through observation or measurements so that the data can be practically useful in solving a problem or such data' [21]. In addition, 'comprehension ability' is defined as 'the ability to discern and understand facts or something based on reasonable judgement and thoughts' [21]. Therefore, taken together, health literacy can be defined as the ability to discern and understand data and knowledge related to mental and physical soundness or robustness.

\section{2) Concepts of health literacy in related disciplines}

The term 'health literacy' was first introduced in 1974, and the word was used in a study by Simonds (1974) upon the impact of health information on the health care system, education system, and the mass media. According to the definition of the American Medical Association, it is a collection of skills needed in a health care environment, such as basic oral reading and arithmetic skills [23,25]. The American Medical Association defines health literacy as "the ability to obtain, process, and understand basic health information and services needed to make appropriate health decisions and follow instructions for treatment" [23]. Therefore, health literacy is individual, social, and cognitive skills that enable individuals to understand and use information to solve their health problems and maintain good health status, and it is a result of typical health education activities [24]. In Korea, several translations for health literacy are interchangeably used, and Korean translations have literal meanings such as 'medical information utilization ability', 'health literacy', and 'health information comprehension ability.' However, there is not yet a single established standard Korean term for health literacy [25]. In addition, as various types of mass media are developed, new capabilities and skills are increasingly required to have health literacy, so the concept of health literacy is sometimes extended to include media literacy or computer literacy [10].

\section{3) Trends in studies on health literacy in nursing and health-} care

In Korea, research on health literacy began to be conducted relatively later compared to other countries and has been carried out mainly in the field of medicine since 2000 [9]. The population groups mainly studied in nursing studies were adults aged 40 years or over and older people aged 60 years or over. 'Health literacy' was once translated into the Korean expression meaning 'medical information comprehension ability', and it has been recently translated into Korean expressions which literally mean 'health literacy', 'health information comprehension ability', or 'health information utilization ability'. In addition, according to the research characteristics, Korean translations with the literal meaning of 'linguistic health information comprehension ability' and 'functional comprehension ability' have also been used [26]. A prior study analyzed the concepts of health literacy presented in studies published in Korea, using Rodgers' evolutionary concept analysis method. This study identified 'information seeking, information understanding, and information utilizing' as the attributes of health literacy, and identified health status, health beliefs, socioeconomic status and information quality as the antecedents of health literacy, and derived the improvement of self-care, improvement of interactions, and reduction of social costs as the consequences of health literacy [18]. The concept of 'oral health literacy' identified in the field of dentistry has been defined and used as 'the degree to which individuals have the capacity to obtain, process, and understand basic health information and services needed to make appropriate oral health decisions' [27]. The concept of 'mental health literacy' was used to assess mental health in married immigrant women, is a concept that applies health literacy to mental symptoms from a social perspective, and it has been defined as the knowledge and beliefs of individuals who are able to recognize and manage their mental health problems such as depression [28]. The term 'e-health literacy', which is a word recently created as a result of the recent development of science and technology, refers to the ability to search, understand, and evaluate health information for solving health problems through the Web or the Internet, and it can be regarded as a concept generated in connection with the social changes due to technological development such as the use of computers and the spread of smart phones [29]. 
4) Antecedents, dimensions, attributes, indicators, and provisional definition of health literacy of patients with cardiovascular disease

The antecedents of health literacy for patients with cardiovascular disease identified in the theoretical stage were physical factors (objective health status, age, and accompanying diseases), emotional factors (health belief, family support, and subjective health status), and social factors (culture, education level, occupation, and economic level). It was found that health literacy for patients with cardiovascular disease can be divided into the individual functional dimension and the interrelational dimension. The individual functional dimension was found to include health information search ability and information utilization skills as well as literacy skills such as reading, writing and numeracy, while the ability to communicate with the medical team was shown to be an attribute of the interrelational dimension. In addition, health literacy for patients with cardiovascular disease has been reported to be closely associated with positive health outcomes and is evaluated to be a predictor of the health status of patients with cardiovascular disease. In short, as health literacy for patients with cardiovascular disease enables patients to recognize their own diseases and understand the medical team's instructions related to the diseases and increases their motivation for health behaviors, health literacy for patients with cardiovascular disease has been reported to lead to successful implementation of health behaviors. Many studies have shown that the implementation of health behaviors are manifested in different forms. In other words, previous studies have demonstrated that the implementation of health behaviors affected medication adherence, which is very important for patients with cardiovascular disease, were manifested in diverse forms of health management, including dietary management such as sodium intake control, stress management, temperance, reduction or cessation of cigarette smoking, and weight control, and influenced communication with the medical team, such as regular visits to medical institutions (Table 1). As a result, in patients with cardiovascular disease, the level of health literacy has been shown to influence the indicators, such as compliance with medical instructions (medication adherence and the improvement of medication compliance), elimination of risk factors (reduction or cessation of smoking, temperance, and weight control), management of influencing factors (changes in eating habits and stress control), and interactions with the medical team (outpatient visits, taking regular examinations, participation in educational programs, participation in immunization programs). In short, health literacy for pa- tients with cardiovascular disease is affected by its antecedents including physical, emotional, and social factors, and it refers to the ability of patients with cardiovascular disease with different levels of literary skills to communicate appropriately with the medical team and to search and use various kinds of health information to maintain and promote health.

\section{Fieldwork Stage}

The participants of this study were adults aged 48 to 85 years with an average age of 67.2 years, and a total of 13 participants (10 males and 3 females) were enrolled in this study. 1 12 years have passed since they were diagnosed with cardiovascular disease. The types of diagnosed diseases were heart failure in 4 persons, myocardial infarction in 3 persons, angina pectoris in 4 persons, atrial fibrillation in 1 person, and arrhythmia in 1 person (Table 2). The attributes of the health literacy of cardiovascular patients derived through the fieldwork was classified into the individual functional and interrelationship dimension. Literacy skills, health information search ability, and health information utilization skills were derived as the attributes of health literacy of the individual functional dimension, while active communication with the medical team and utilization of health information support resources were derived as the attributes of health literacy of the interrelational dimension.

\section{1) Individual functional dimension}

(1) Literacy skills

'Literacy skills' refers to not only the oral reading ability but also the ability to extract the meanings of text. In other words, its concept encompasses prose literacy to understand words or instructions, and it refers to the ability to apply one's literacy skills appropriately to understand prescriptions, instructions, or signs, make an appointment to see the doctor, or do some other things. Because of the characteristic of Hangul, the Korean alphabet, which allows people to pronounce words even when they do not know their meanings, many of the participants stated that they did not understand the meanings of words or instructions, although they had no difficulty reading. Among the participants, one older person (83 years of age) was illiterate.

I don't know the names of medicines well. I don't understand what they mean even if I read words on the paper. Does it say what I must not take it with?. (Participant 1) 
If I see some materials where a lot of words are written, I can't understand them. Sometimes, if there are some pictures, it's a little easier to understand. (Participant 7)

The consent form consisted of two sheets of paper. They were very long and wordy, but I couldn't understand them. The document seemed to say that I may die if something goes wrong during the procedure, but I couldn't clearly understand the content, so I just smiled awkwardly. (Participant 13)

(2) Health information search ability

'Health information search ability' refers to the ability to collect and identify information related to cardiovascular disease, and it means the ability to access and extract necessary information using multiple means and diverse resources. The participants said that they obtained information through various channels such as mobile phones, computers, brochures, guides, and book, and many of them mentioned that they searched for information about the symptoms, treatments, and medications using their mobile phones.

When the doctor first said I had angina, I didn't know what it was. So I searched it on my mobile phone. I was so scared. (Participant 6)

If I hear of any TV program or show about the heart, I try to find and watch it. A TV program called the Mysteries of the Human Body also broadcasted a program on heart disease. I watched it, too. Manmulsang, a TV program, also aired a program on the heart, and they say that people may die of this disease. (Participant 2)

When I searched information at Naver, I found an Internet cafe. People have uploaded information such as good and experienced doctors and ways to cure disease and they are sharing their experiences about their diseases, so I joined the cafe. (Participant 9)

(3) Health information utilization skills

'Health information utilization skills' means the ability of individuals to discern and select necessary information among diverse information obtained through various channels to make appropriate health-related decisions on their own and implement health behaviors according to appropriate health information.

There are so many TV programs related to the heart health or heart disease, so I don't know if what they say in the programs is always correct. They say
Omega 3 is good for your heart on home shopping channels, but it's just part of the sales pitch, isn't it? They're just trying to sell their products, aren't they? Actually, I don't know what they say is really true or false, but you could give it a try since it is said to be good for your health. (Participant 5)

I feel I keep putting on weight since I don't work, so I try to eat a lot of vegetables and fruits, and I occasionally check my body weight. Since they say if you become fat, it's dangerous, I try to eat less salty food and not to drink or smoke. But they are the hardest things to put into practice. (Participant 12)

\section{2) Interrelational dimension}

(1) Active Communication with the Medical Team

'Active Communication with the Medical Team' refers to the communication ability to describe one's symptoms and physical and mental conditions to the medical team in detail and clearly and to ask for easy and clear explanations if the instructions or explanations of the medical team are not understood. There were significant differences in the ability to communicate with the medical team according to the individuals' age, education level, and accompanying diseases, and these differences reflected Korean culture of medical treatment and its characteristics.

When I received the stent procedure, the doctor gave me some explanations and told me to sign a document, but I couldn't understand what he said. Because they said that if I didn't receive the treatment quickly, I might die, I signed it, but I honestly had no idea what was written in the document. (Participant 1)

At first, I didn't understand what the doctor said, so I just answered yes. I had no idea of what a stent was or what a balloon was. I think what the doctor said was that it was narrowed or blocked because of fat and it was necessary to open it.(Participant 13)

Even if I want to ask a question again, I can't do that because the nurses are too busy. And I feel sorry and awkward when I try to ask the same question again or ask for the same explanation many times. (Participant 7)

(2) Utilization of health information support sources

The participants stated that they were able to understand the medical team's instructions through the information obtained through a mobile phone or the media, their family's help, and explanations of other people around them. Many participants said that it was difficult to communicate with the medical team during a short treatment time, and that they selectively acquire necessary 
information through individual information searches after the treatment. In the process of selecting information, the most important factor in selectively acquiring diverse kinds of health information was found to be the family or the cohabitant, who influence the patients' daily life, such as lifestyle modification and medication adherence.

The doctor sees me for about one minute. After waiting so long, I see the doctor only for a minute. Then, I remember everything the doctor said? No, I can't remember. When I go with my children, they explain to me what the doctor said again. And what they say on TV seems to be easier to understand. (Participant 5)

All my family are making a fuss about my disease. My kids wrote a list of foods on a piece of paper and put it on the door of the refrigerator. The kinds of foods I should eat. They say I should eat such foods. My wife also cares about my diet, saying that I should eat a lot of vegetables as well as nuts such as walnuts and almonds. (Participant 3 )

I try to remember to take medicine on time. Because the doctor said if I don't take medicine, my blood vessels and arteries may get blocked again and I may die. What do people like us know about disease or medicine? Just because they say if I don't take it, I may die, I just try to take medicine regularly. As soon as I wake up every morning, my wife reminds me to take medicine. (Participant 13)

\section{Final Analysis Stage}

In the final analysis stage, the investigator conducted a comprehensive analysis of the results of the systematic literature review and field research through discussions to clearly identify the definition, attributes and indicators of health literacy for patients with cardiovascular disease. In the theoretical stage, four attributes of two dimensions were identified, and in the fieldwork stage, five attributes of two dimensions were derived. The 'ability to communicate with the medical team,' which was derived as an attribute of the interrelational dimension in the theoretical stage, was expanded to two attributes, 'active communication with the medical team' and 'utilization of health information support resources', in the fieldwork stage. As a result, in the final analysis stage, two dimensions and five attributes were derived by integrating the theoretical and fieldwork stages (Table 3). Literacy skills, health information search ability, and health information utilization skills were derived as the attributes of the individual functional dimension, and active communication with the medical team and utilization of health information support resources were derived as the attributes of the interrelational dimension. Based on the attributes identified in the theoretical and fieldwork stages, health literacy for patients with cardiovascular disease can be defined as individuals' individualized and integrated ability to explore and utilize a variety of health information needed to make

Table 3. Dimensions, Attributes, Indicators of Health Literacy of Patients with Cardiovascular Disease in Final Stage

\begin{tabular}{|c|c|c|}
\hline Dimensions & Attributes & Indicators \\
\hline \multirow[t]{3}{*}{$\begin{array}{l}\text { Individual } \\
\text { functional }\end{array}$} & Literacy skills & $\begin{array}{l}\text { - Read and write to understand; read and understand prescriptions, pharmacology, } \\
\text { doctor's appointments, manuals, and notices. } \\
\text { - Understanding and signing the consent form relating to treatment }\end{array}$ \\
\hline & $\begin{array}{l}\text { Health information } \\
\text { search ability }\end{array}$ & $\begin{array}{l}\text { Collect information related to cardiovascular disease } \\
\text { Search information through TV cell phones, computers, brochures, and books. } \\
\text { Searching for symptoms, treatments, and drug information with your mobile } \\
\text { phone }\end{array}$ \\
\hline & $\begin{array}{l}\text { Health information } \\
\text { utilization skills }\end{array}$ & $\begin{array}{l}\text { Select the required information from the various information. } \\
\text { - Have faith in acquired health information. } \\
\text { - Decided to act for health according to health information. } \\
\text { - Utilizing information for health }\end{array}$ \\
\hline \multirow[t]{2}{*}{ Interrelational } & $\begin{array}{l}\text { Active communication } \\
\text { with the medical team }\end{array}$ & $\begin{array}{l}\text { - Explain symptoms and physical condition to the clinical health workforce } \\
\text { - To ask about treatment instructions. } \\
\text { - To ask again about words that are not understood }\end{array}$ \\
\hline & $\begin{array}{l}\text { Utilization of health } \\
\text { information support } \\
\text { resources }\end{array}$ & $\begin{array}{l}\text { - To understand treatment instructions with the help of the family or cohabitant. } \\
\text { - Trying to change one's lifestyle with the help of a family member or cohabitant. } \\
\text { Establish a treatment plan with your family. } \\
\text { - Understanding of cardiovascular disease through mobile phones and media }\end{array}$ \\
\hline
\end{tabular}


appropriate health-related decisions, actively communicate with the medical team, and utilize supportive resources in order to lead a healthy life during a chronic course of disease after the diagnosis of cardiovascular disease.

\section{DISCUSSION}

The health literacy of patients with cardiovascular disease is not simply the ability to read and understand but the complex and multidimensional ability of individuals as smart patients [30] who actively participate in the disease treatment process in the healthcare environments changing into consumer-centered ones, and it includes a positive direction toward the implementation of self-care. Therefore, this study aimed to identify the attributes reflecting the characteristics of Koreans by applying the hybrid model method of performing a systematic review of the literature in conjunction with interviews with community-dwelling patients with chronic cardiovascular disease.

In this study, health literacy of Korean patients with cardiovascular disease was classified into individual functional and interpersonal relational factors, and five attributes and indicators were derived. In previous studies of health literacy of patients with cardiovascular disease conducted from Western perspectives, attributes of health literacy were identified, focused on individuals' literacy skills, numeracy, and reading comprehension, and the attributes of health literacy associated with the social system, culture, and family support were partially included in those studies $[6,13]$. Considering such limitations of previous studies, the results of this study are believed to represent meaningful progress from existing studies.

In Korea, the measurement of health literacy has been made on linguistic health literacy regarding understanding health-related terms and functional health literacy regarding the ability to understand or practice health-related behaviors or instructions [25]. Even in studies on health literacy of Korean patients with cardiovascular disease [6,13], either KHLAT [8] or KFHLT [9] was used to measure health literacy. The two tools measure different aspects of health literacy; in other words, one measures the vocabulary ability of linguistic literacy and the other assesses the comprehension ability of functional literacy. Thus, each of the tools does not comprehensively measure the overall aspects of integrated health literacy of patients with cardiovascular disease. In this regard, previous studies did not take into consideration the Korean medical culture, conditions of physicians' offices in Korea, and disease characteristics and ages of patients with cardiovascular disease, so there is a need to comprehensively analyze the overall aspects of health literacy. In particular, a majority of patients with cardiovascular disease are older people, and elderly patients with cardiovascular disease have poor oral reading skills because of senile infirmity and also have poor signification skills. Previous studies have shown that these patients with low literacy often have characteristics that hinder smooth interactions with the medical team [23]. It has been frequently reported that people with low literacy know their lack of literacy, are ashamed of the fact and tend not to let others know, and it has also been reported that they do not disclose this fact to their spouses or children and that they hardly reveal it to the doctor or nurse [10]. This feeling of shame and lack of confidence are barriers to the success of treatment, and they are very likely to prevent patients from asking for clear explanations to understand medications or treatment instructions. The participants of this study also stated that even though they did not understand treatment instructions or guidances, they did not bring themselves to ask questions due to the authoritative attitude of the medical team, short treatment time or the fear that they might disclose their low level of health literacy. In addition, authoritative attitudes of the medical team and negative experiences in the treatment implementation process were manifested as dissatisfaction with the medical team. This attribute is considered to be a result reflecting the unique medical culture and situations of Korea. Given that the consultation time per patient in general hospitals in Korea is scheduled and managed in units of minutes, it is highly unlikely that the medical team checks whether patients properly understand their instructions. As a result, patients with low health literacy are not expected to receive additional explanations or repeated instructions from the medical team. These findings suggest that nurses need to assess health literacy for patients with cardiovascular disease during outpatient visits for disease management.

Literacy skills, health information search ability, and health information utilization ability identified as the attributes of the individual functional dimension in this study were similar to the three attributes of information seeking, information understanding, and information utilization which Kim et al. [18] suggested through the concept analysis for the general population in Korea. However, in this study, the interrelational factors, active communication with the medical team and utilization of health information support resources, were additionally identified through the actual interviews with the participants in the fieldwork stage by applying the hybrid model method. 
These are considered to be appropriate and desirable attributes considering that the current medical culture pursues and is moving toward 'smart patients,' emphasizing the right of patients to actively participate in the process of disease treatment. In an interview with a patient with myocardial infarction, it was found that the patient did not understand the doctor's explanations when filling out the consent form for the stent procedure and was able to understand the contents only after obtaining additional explanations from the family. The participants' statements during interviews revealed that even if the patient acquires information related to cardiovascular disease from the media or through a mobile phone, that the family, caregiver, and cohabitant who spend time with the patient have a significant influence on the patient's selection and utilization of the information. This finding demonstrated that the use of health information support resources is an important attribute of health literacy. In this study, the use of health information support resources was derived as an attribute of the interrelational dimension. Most of the elderly patients or patients with low health literacy received external support from the family such as children and the spouse, the caregiver, or the cohabitant. They played the roles of explaining the health information that the patient did not understand using easier expressions, asking questions instead of the patients, and reminding them to increase compliance with treatment. In addition, in the case of patients with low health literacy, their family members were found to search and access health information instead of the patients, provide health information, and also play an important role in providing motivation related to health behaviors. The participants also stated that they made health-related decisions with the help of their families and that their family encouraged them to perform health behaviors. Based on the statements of the participants, it is thought that while health literacy of the functional dimension can be regarded as each individual's ability, health literacy of the relational dimension can be seen as the ability combined with some sociocultural elements. In this regard, it is expected that those who are vulnerable in terms of health information support resources are more likely to lack the ability to understand health information at the relational dimension. The results of this study described above suggest that an assessment of the existence and use of supporting resources, including the family, should be first conducted in order to induce effective selfcare of the patients.

In this study, the communication ability of the interrelational dimension means active two-way communication between the medical team and patients with cardiovas- cular disease. According to a previous study, patients with low health literacy are likely to have great difficulties in explaining their symptoms. It has been reported that the low literacy level is closely associated with the limitation of language expressions, and that patients with low literacy are not able to communicate their symptoms and medical histories logically and consistently [10]. This problem is likely to result in difficulty in communication due to the medical team's different expression methods and sentence structures. This difficulty in communication may lead to a variety of consequences such as the decrease of recognition of accurate symptoms, lower accuracy of diagnosis, and lower satisfaction with treatment. Therefore, the medical team should provide patient-oriented education that focuses on the health literacy levels of patients so that Korean patients with cardiovascular disease accurately recognize their disease status and autonomously select optimal medical services as smart patients rather than passively implementing the medical team's instructions.

The strength of this study is that we not only identified the attributes of the cognitive and functional dimension of health literacy of Korean patients with cardiovascular disease, such as individuals' literacy skills, health information search ability, and health information utilization skills, but also included the attributes of the interrelational dimension, such as active communication with the medical team and utilization of health information support resources as the important attributes related to Korean culture by conducting interviews with actual patients in the field research. These results of this study are expected to contribute to the establishment of supportive nursing interventions to prevent the recurrence and exacerbation of cardiovascular diseases and to establish the basis for establishing and spreading the consumer-centered medical culture of smart patients. However, the indicators derived in this study may have limitations because this study was focused on in-depth interviews with cardiovascular patients rather than clinical observations by the medical team in order to derive health literacy in consideration of the chronic disease characteristics of cardiovascular patients and individuals' disease perception.

\section{CONCLUSION}

As a result of analyzing the concept of health literacy of Korean patients with cardiovascular disease by applying the hybrid model, two dimensions and five attributes of the health literacy were derived. In other words, literary skills, health information search ability and health information utilization skills were identified as the attributes of 
the individual functional dimension, and active communication with the medical team and utilization of health information support resources were derived as the attributes of the interrelational dimension. Health literacy was defined as the individualized and integrated ability to explore and utilize diverse health information needed by individuals with different levels of literacy skills and different degrees of supportive resources to make appropriate health-related decisions, to have active communication with the medical team, and utilize supportive resources in order to lead a healthy life during a chronic course of disease after the diagnosis of chronic cardiovascular disease. In the future, based on the attributes and indicators derived from the concept analysis in this study, research should be conducted to develop measurement tools with high validity and reliability to assess the health literacy of Korean patients with cardiovascular disease. In addition, there is a need to conduct a follow-up study to evaluate the usefulness of the concept of health literacy of patients with cardiovascular disease and to explore its relationship with various outcome indicators.

\section{REFERENCES}

1. Korea Centers for Disease Control and Prevention. Chronic disease current status and issues 2017, chronic illness factbook [Internet]. Cheongju: Korea Centers for Disease Control and Prevention. 2018 [cited 2019 May 1]. Available from: http://www.cdc.go.kr/gallery.es?mid=a40303020100\&bid= 0003\&act=view\&list_no $=136662$

2. Centers for Disease Control and Prevention. Indicator definitions cardiovascular disease [Internet]. Atlanta: Centers for Disease Control and Prevention; 2017 [cited 2019 June 10]. Available from:

https://www.cdc.gov/cdi/definitions/cardiovascular-disease .html\#CVD1_1

3. Bae YH, Lee KW. Risk factors for cardiovascular disease in adults aged 30 years and older. Journal of the Korean Society of Integrative Medicine. 2016;4(2):97-107. https://doi.org/10.15268/ksim.2016.4.2.097

4. Lee MS. Health literacy and health behaviors among older adults with cardio-cerebro vascular disease residing in rural areas. Korean Journal of Adult Nursing. 2017;29(3):256-265. https://doi.org/10.7475/kjan.2017.29.3.256

5. Kim SH, Lee SH. Influences of knowledge, self-efficacy, and social support on sick role behavior in patients with coronary artery disease. Journal of Korean Public Health Nursing, 2014; 28(2):228-240. https:// doi.org/10.5932/jkphn.2014.28.2.228

6. Jung EY, Hwang SK. Health literacy and health behavior compliance in patients with coronary artery disease. Korean Jour- nal of Adult Nursing. 2015;27(3):251-261.

https://doi.org/10.7475/kjan.2015.27.3.251

7. Jeong JH, Kim JS. Health literacy, health risk perception and health behavior of elders. Journal of Korean Academy Community Health Nursing. 2014;25(1):65-73.

https://doi.org/10.12799/jkachn.2014.25.1.65

8. Kim SS, Kim SH, Lee SY. Health literacy: Development of a Korean health literacy assessment tool. Korean Journal of Health Education and Promotion. 2005;22(4):215-227.

9. Kim SH, Lee E. The influence of functional literacy on perceived health status in Korean older adults. Journal of Korean Academy Nursing. 2008;38(2):195-203.

https://doi.org/10.4040/jkan.2008.38.2.195

10. Schwartzberg JG, VanGeest J, Wang C. Understanding health literacy implications for medicine and public health. Chicago: AMA Press; 2005. 253 p.

11. Paek KS. Impact of health literacy on adherence to self care behavior among the elderly with hypertension in a community. Crisisonomy. 2015;11(8):157-174.

12. An JS, Yang SJ. Development of a health literacy assessment scale for asian immigrant women in South Korea. Journal of Korean Academy of Community Health Nursing. 2015;26(4): 330-341. https://doi.org/10.12799/jkachn.2015.26.4.330

13. Sin KM, Chu SH, Jang YS, Kang SM. Relationships between health literacy, knowledge and self-care behaviors in elderly patients with heart failure. Journal of Korean Clinical Nursing Research. 2016;22(1):28-37.

https://doi.org/10.22650/JKCNR.2016.22.1.28

14. Davis TC, Long SW, Jackson RH, Mayeaux EJ, George RB, Murphy PW, et al. Rapid estimate of adult literacy in medicine: A shortened screening instrument. Family Medicine. 1993;25 (6):391-395.

15. Parker RM, Baker DW, Williams MV, Nurss JR. The test of functional health literacy in adults: A new instrument for measuring patients' literacy skills. Journal of General Internal Medicine. 1995;10(10):537-541.

https://doi.org/10.1007/bf02640361

16. Lee TW. Development and evaluation of health literacy improvement program for the low income elderly with hypertension. Research Report. Seoul: Yonsei University \& Korea Foundation for the Advancement of Science \& Technology; 2010. Report No.: 2007-0053325.

17. De Gagnea, Jennie C, Oh JA. Health promotion education in a virtual world: A concept analysis. The Journal of Korean Academic Society of Nursing Education. 2012;18(1):81-94. https://doi.org/10.5977/jkasne.2012.18.1.081

18. Kim SE, Oh JN, Lee YM. Health literacy: an evolutionary concept analysis. The Journal of Korean Academic Society of Nursing Education. 2013;19(4):558-570.

https://doi.org/10.5977/jkasne.2013.19.4.558 
19. Schwartz-Barcott D, Kim HS. An expansion and elaboration of the hybrid model of concept development. In: Rodgers BL, Knafl KA, editors. Concept development in nursing: foundations, techniques, and applications. 2nd ed. Philadelphia (PA): Saunders; 2000. p. 129-159.

20. Strauss AL, Corbin JM. Basics of qualitative research: grounded theory procedures and techniques. Newbury Park, CA: Sage; 1990.270 p.

21. National Institute of Korean Language. Standard Korean language dictionary [Internet]. Seoul: National Institute of Korean Language; 2019 [cited 2019 Jun 1]. Available from: https://stdict.korean.go.kr/search/searchResult.do?pageSize $=10 \&$ searchKeyword=\%EA\%B1\%B4\%EA\%B0\%95

22. Kim YS, Park BH, Lee HY. A study on predicting health literacy of Korean elderly using Andersen's health behavior model. Journal of Welfare for the Aged Institute. 2014;65(1):35-57.

23. Son YJ, Kim SD, Jang HJ, Yun YR, Kim HM, Park JI, et al. Factors influencing health literacy in community-dwelling adults. Korean Journal of Health Promotion. 2012;12(2):100-108.

24. Mark K, Elizabeth G, Ying J, Christine P. The health literacy of America's adult: results from the 2003 national assessment of adult literacy. Washington DC. U.S Department of Education,
2006 September. Report No.: NCES 2006-83.

25. Lee SM, Kim SY, You KS, Seo DC. Review on health literacy and application in pharmacoepidemiology. Korean Society for Phamacoepidemiology and Risk Management. 2016;8(1):8-18.

26. Park HJ, Hwang SK. Linguistic and functional health literacy among community-dwelling old adults. Global Health and Nursing. 2014;4(2):49-58.

27. Lee HJ, Chang KW. Oral health literacy of Korean adults. Journal of the Korean Academy of Dental Health. 2017;41(2): 102-109. https://doi.org/10.11149/jkaoh.2017.41.2.102

28. Park BH, Kim HJ, Kim YS. A study on mental health literacy and social relationship among marriage migration females. Korean Association of Health and Medical Sociology. 2016;42: 111-135

29. Lee SS, Son HJ, Lee DS, Kang HW. The Influence of e-health literacy, subjective health status, and health information seeking behavior on the internet on health promoting behavior. Korean Society for Wellness. 2017;12(4):55-67. https://doi.org/10.21097/ksw.2017.11.12.4.55

30. Roizen M, Oz M. You: the smart patient. New York: Simon \& Schuster; 2006. 578 p. 


\section{Appendix. List of Studies Included in Conceptual Analysis}

1. Williams AM, Andrew S, Paul W, Andre T, Gillian PR, Anita $\mathrm{M}$, et al. Characteristics of people with low health literacy on coronary heart disease GP registers in South London: A crosssectional study. BMJ Open. 2013;3:e001503. https://doi.org/10.1136/bmjopen-2012-001503

2. Sue C, Janet S, Margaret K, Michelle L, Susan R, Joanne L, et al. Cardiovascular disease medication health literacy among indigenous peoples: Design and protocol of an intervention trial in indigenous primary care services. BMC Public Health. 2014;14(1):714. https://doi.org/10.1186/1471-2458-14-714

3. Aaby A, Friis K, Christensen B, Rowlands G, Maindal HT. Health literacy is associated with health behaviour and self-reported health: A large population-based study in individuals with cardiovascular disease. European Journal of Preventive Cardiology. 2017;24(17):1880-1888. https://doi.org/10.1177/2047487317729538

4. June C. Health literacy and health status in people with chronic heart failure. Clinical Nurse Specialist. 2018;32(1):29-42 https://doi.org/10.1097/NUR.0000000000000346

5. Ko MS, Kang KJ. Influence of health literacy and health empowerment on health behavior practice in elderly outpatients with coronary artery disease. Journal of Korean Clinical Nursing Research. 2018;24(3):293-302. https://doi.org/10.22650/JKCNR.2018.24.3.293

6. Noureldin M1, Plake KS, Morrow DG, Tu W, Wu J, Murray MD. Effect of health literacy on drug adherence in patients with heart failure. Pharmacotherapy. 2012;32(9):819-826. https://doi.org/10.1002/j.1875-9114.2012.01109.x

7. Wu JR, Reilly CM, Holland J, Higgins M, Clark PC, Dunbar SB. Relationship of healthliteracy of heart failure patients and their family members on heart failure knowledge and self-care. Journal of Family Nursing. 2017;23(1):116-137. https://doi.org/10.1177/1074840716684808 